\title{
Hysteresis in mesoscopic superconducting disks: the Bean-Livingston barrier
}

\author{
P. Singha Ded, V. A. Schweigert因, and F. M. Peeters \\ Department of Physics, University of Antwerp (UIA), B-2610 Antwerpen, Belgium.
}

\begin{abstract}
The magnetization behavior of mesoscopic superconducting disks can show hysteretic behavior which we explain by using the Ginzburg-Landau (GL) theory and properly taking into account the de-magnetization effects due to geometrical form factors. In large disks the Bean-Livingston surface barrier is responsible for the hysteresis. While in small disks a volume barrier is responsible for this hysteresis. It is shown that although the sample magnetization is diamagnetic (negative), the measured magnetization can be positive at certain fields as observed experimentally, which is a consequence of the de-magnetization effects and the experimental set up.
\end{abstract}

PACS numbers: 74.25.Ha; 74.60.Ec; 74.80.-g

Hysteresis in the magnetization of superconductors [1] 3] is a fascinating field of fundamental research which is related to the occurrence of meta-stability. Here we will investigate this phenomenon in single mesoscopic superconducting disks. Recently, Geim et al [4] used the Hall probe technique to study the magnetization of single mesoscopic $A l$ disks. The investigated disks can be classified as few fluxoid disks (FwFD) and fractional fluxoid disks (FrFD). The FrFD are so tiny that fluxoids cannot nucleate in them because the required magnetic field to create a fluxoid in it exceeds the critical field of the sample. The FwFD are those in which a few fluxoids can nucleate (typically of the order of 10 and definitely greater than 1) before it makes a transition to the normal state. It was found that these disks: 1) exhibit a variety of phase transitions (type I or type II) that are absent in macroscopic samples; 2) show strong hysteresis behavior, and 3 ) in the field down sweep can exhibit paramagnetic behavior.

In our earlier work [5] we presented a quantitative explanation of the magnetization of the different disks as function of increasing external magnetic field without using any fitting parameters. In increasing magnetic field the experimental data were found to follow the magnetization of the ground state of the sample. Axially symmetric solutions with a fixed angular momentum $L$ were assumed and the non-linear GL eqns. were solved for disks with a finite thickness. Switching between the different $L$ states occurs when their free energies are equal leading to steps in the magnetization of the mesoscopic superconductor. In increasing field the Bean Livingston (BL) surface barrier responsible for meta-stability, is destroyed by boundary roughness which explains why the system follows the ground state. The BL barrier arises from the fact that the superconducting currents around a vortex is in the opposite direction to the screening cur- rents at the surface of the sample which does not allow the nucleation of vortices at the boundary, although the free energy is lowered when the vortex moves to the center of the sample.

The decreasing field behavior of the magnetization, which was not studied in our previous work, can be very different and can even show paramagnetic behavior. One of the reasons for this different behavior is that the Bean Livingston barrier in this case is not destroyed by surface defects and the system will not evolve along the ground state. A simple estimate of the BL barrier using the approach outlined in Ref. [3] shows that it can be several orders of magnitude larger than $k T$ for experiments done at $0.4 \mathrm{~K}$. In this case the steady state will not necessarily be the ground state but will be an excited state determined by the history of the sample and the meta-stability created by the Bean Livingston barrier.

Our theoretical approach was outlined in in Refs. [5, 60. The order parameter is considered to be uniform in the $\mathrm{z}$ direction which is a very good approximation for thin disks, i.e., thickness less than the coherence length. We use the Gauss-Seidel method to solve the non-linear GL eqn. (Eqn. (1) in Ref [5]) and the fast Fourier transform to solve the 3D Maxwell eqn. (Eqn. (2) in Ref. [5]). The order parameter of the previous magnetic field is taken as the initial order parameter for a particular magnetic field. This ensures that the system does not escape from the barriers leading to meta-stability and converges differently in increasing and decreasing fields. A large number of iterations (typically of the order of $5 \times 10^{4}$ ) are then made to arrive at a self consistent solution.

As a typical case let us consider a $\mathrm{FwFD}$ (radius $R=0.8 \mu \mathrm{m}$, thickness $d=0.134 \mu \mathrm{m}$, coherence length $\xi(0)=0.183 \mu \mathrm{m}$ and penetration length $\lambda(0)=0.07 \mu \mathrm{m})$ with parameters comparable to one of the disks used in the experiment [ 4 . The solution assuming axial symmetry is referred to as the 2D solution (due to the symmetry the azimuthal direction drops out) whereas the general solution without assuming axial symmetry will be referred to as $3 \mathrm{D}$ solution. Let us consider the $2 \mathrm{D}$ solution first. The dimensionless free energy $G$, in units of $H_{c}^{2} V / 8 \pi$ [5] (here $\mathrm{V}$ is the volume of the sample), is shown in Fig. 1(b) by the thin solid curves as a function of the applied magnetic field for the different $L$ states. The corresponding magnetization for these different $L$ states is shown in Fig. 1(a) by the thin solid curves. Hence from Fig. 1(b), it can be seen that up to a magnetic field of 42.6 Gauss, the $L=0$ state is the ground state. Beyond this field the $L=1$ state becomes the ground state. As we increase the field, the higher $L$ states become the lowest energy state. This continues as long as the free energy 
$\mathrm{G}$ is negative. A positive $\mathrm{G}$ means that the normal state has a lower free energy than the superconducting state and the superconductor turns normal. The free energy of the ground state of the system is therefore given by the thick solid curve in Fig. 1(b). The corresponding magnetization along this ground state is given by the thick solid curve in Fig. 1(a). However the free energy and the magnetization in increasing magnetic field as given by the $3 \mathrm{D}$ solution is given by the thick dotted curve in Fig. 1. Thus the 3D solution in increasing magnetic field, takes the system along a steady state that conserves $L$ up to the point where the free energy is zero at which point a jump to a higher $L$ state occurs. The free energy and magnetization curve in decreasing magnetic field as given by the $3 \mathrm{D}$ solution is given in Fig. 1 by the thick dashed curves. It can be seen that along the steady state the system moves along states whose free energy is much higher than that of the ground state.

The experimental data for the disk considered in Fig. 1, in decreasing and increasing field are given in Fig. 2 by open circles and squares, respectively. These curves are plotted according to the scale on the left y-axis. The dashed and dotted curves in Fig. 2 are the same as the thick solid and thick dashed curves in Fig. 1(a), their scale being depicted on the right $y$-axis. The magnitude of the measured magnetization (open circles and squares) is clearly too small compared to the sample magnetization in increasing and decreasing fields (dashed and dotted curves). Furthermore in the experimental data, paramagnetic behavior is found for certain magnetic fields. Although diamagnetism is a fundamental property of superconductors, previously a paramagnetic Meissner effect [7] was observed on large Nb disks. This discovery lead to intensive research but the effect is still not completely understood 8]. In the presence of pinning also superconducting samples can exhibit paramagnetic behavior. But we found that this discrepancy (i.e., the factor of 25 and the paramagnetic behavior) can be explained by considering the full experimental set up of Ref. [4.

The magnetometry used in the experimental work of Ref [4] is explained in detail in Ref. [9]. The superconducting sample is mounted on top of a small ballistic Hall cross and the magnetization of the superconducting disk was measured through the Hall effect. In Ref. [10] it was shown that the Hall voltage of a Hall cross, in the ballistic regime, is determined by the average magnetic field piercing through the Hall cross region. The Hall cross has a larger area than the sample and it measures the magnetization of this area rather than the magnetization of the sample. The effective area of the Hall cross is approximately $3 \mu m \times 3 \mu m$. The field distribution in case of thin disks is extremely non-uniform inside as well as outside the disks and the detector size will have an effect on the measured magnitude of the magnetization, the nature and extent will depend on the magnetic field profile outside the sample. We calculate the magnetization measured by the detector by integrating the field expelled from its area, i.e., the Hall cross. We took the detector to be a square whose side is of length $3.1 \mu \mathrm{m}$ and is placed just below the superconducting disk. The resulting magnetization is given by the solid curves in Fig. 2 drawn according to the scale on the left y-axis i.e., with the same scale as the experimental data. Note that by including the effect of the detector, the magnetization 1) is scaled down considerably, 2) the line shape is changed slightly, and 3) the detector output can give a positive magnetization although the sample itself is diamagnetic. Note that these three factors bring the theoretical result very close to the experimental result which explains even the apparent diamagnetic behavior. Only at the position of the last jump there is a noticeable difference. This may be due to some pinning center near the center of the disk that becomes effective when the giant vortex state shrinks to the center. We found that decreasing the detector to an area enhances the apparent paramagnetic behavior. In the inset of Fig. 2 we compare the magnetization for a detector size of $2 \mu m \times 2 \mu m$ (dotted curve) with that for a detector size of $3.1 \mu \mathrm{mx} 3.1 \mu \mathrm{m}$ (solid curve).

In order to explain the reason why a larger detector can result in an apparent paramagnetic behavior we show in Fig. 3 the total magnetic field (applied field plus the field due to the magnetization of the sample) along a line passing through the center of the disk. Note that far away from the disk region the field is equal to the applied field which is 59.65 Gauss and which is a field where the detector magnetization shows a paramagnetic behavior. In the center of the disk the magnetic field is much larger than the applied field because of the flux trapped by the BL barrier. Hence the central region is paramagnetic. Near the edge of the disk, the magnetic field is much smaller than the applied field and is thus a diamagnetic region. It is this region where superconductivity is maximum and the disk is in the giant vortex state. The sample magnetization is the resultant magnetization of these two regions, and the total magnetization turns out to be diamagnetic. But when we calculate the detector magnetization, we have to include the magnetic field in the region outside the disk which is strongly paramagnetic. The magnetic field just outside the disk is larger than the applied field because of the strong flux expulsion from the disk and the important de-magnetization effects in finite thickness disks. Or in other words in this region the magnetization due to the currents flowing in the disk is added to the external field and both are in the same direction. The $2 \mu \mathrm{m}$ detector show greater paramagnetism that the $3 \mu \mathrm{m}$ detector due to the fact that over a very large area the net flux expelled should be zero (magnetic field far away from the sample decreases as $1 / r^{3}$ and area of a detector increases as $r^{2}$. Consequently magnetization will disappear as $\left.1 / r^{5}\right)$. However, for sufficiently small detectors the paramagnetic behavior will disappear when its size becomes comparable to the sample size. In the experiment of Thompson et al [7], a paramagnetic Meissner effect was observed when $\mathrm{Nb}$ disks were cooled down from their normal state to the superconducting state, in the presence and the absence 
of an external magnetic field. The paramagnetic field outside the sample due to the diamagnetic currents inside the sample will be large for those large disks $(\mathrm{R}=3.2$ $\mathrm{mm}$ ). Hence our explanation may also be relevant for their experiment.

From the above discussion of the magnetic field distribution in and around the superconducting disk one may ask the question whether one can observe the superconductor in a state such that the paramagnetic region at the center has a larger contribution than the diamagnetic region at the boundary [8]. In this case the sample itself would be paramagnetic. Indeed when the thickness of the sample is greatly reduced, the sample magnetization itself can be paramagnetic in a field down sweep. We reduced the thickness by a factor of 10 and kept all other parameters fixed which lead to the sample magnetization as shown in the inset of Fig. 3. The maximum paramagnetism is $0.027 \mathrm{G}$ which occurs at $13 \mathrm{G}$ and which is indicated by a small circle in the figure. For such a thin disk, the diamagnetic response is very small and the flux trapped inside the giant vortex state determines the sign of the response. The magnetic field distribution for this case is given in Fig. 3 by the dotted curve. One can easily notice the weak flux expulsion from the diamagnetic boundary and as a result the paramagnetic region outside the sample is negligible.

Next we consider a FrFD (radius $R=0.5 \mu m$, thickness $d=0.15 \mu \mathrm{m}$, coherence length $\xi(0)=0.25 \mu \mathrm{m}$ and penetration length $\lambda(0)=0.07 \mu m$ ) with a larger coherence length than the previous one, which is probably due to smaller disorder in the disk. The disk shows (see Fig. 4) a first order phase transition to the normal state. Its behavior in increasing field was explained in Ref. [5]. Hysteresis in case of this FrFD is novel because the BL barrier cannot result in meta-stability here. The BL barrier requires the existence of a vortex (which is not present inside the disk) and opposite flowing currents inside the sample. In the following we give an explanation for this hysteresis. For the FrFD we use the 2D solution again, because of its high accuracy and because it is correct in the absence of multiple vortices. The experimental data in increasing and decreasing fields are shown in Fig. 4 by the squares and circles, respectively. The $2 \mathrm{D}$ solution in increasing and decreasing fields are given by the dashed and solid curves, respectively, where the detector size is included in our calculations. We are using a square detector of width $2.9 \mu \mathrm{m}$ placed just below the sample. Although the position of the jump in decreasing field does not coincide with the experiment the non-linear line shape and magnitude of the magnetization is nicely reproduced. A straight dotted line is drawn tangent to the experimental data at the origin as a guide to the eye in order to accentuate this non-linear behavior. The origin of this hysteresis is due to meta-stability created by a volume barrier and not the BL surface barrier. In the present case of a first order transition, the free energy has two local minima which correspond to two different values of the order parameter which correspond to the normal and superconducting states, respectively. These two minima are separated by a maximum which acts as a barrier when the system tries to switch from one minimum to the other at the critical point. To differentiate it from the surface barrier we call it volume barrier. As this barrier only appears near the critical field for each $L$ state, this barrier is not relevant for the FwFD where transitions between different $L$ states occur well below the critical field for them. It is true that in decreasing field the position of the jump does not coincide with that of the experiment but this position is extremely dependent on small fluctuations in the normal system where the order parameter is zero. The order parameter has to jump from zero to a large value which is difficult in the absence of some external nucleation center. If the order parameter starts growing in some region of the sample, the neighboring regions tries to destroy it because that will reduce the gradient term in the Free energy. Although if the order parameter at every point is large then the free energy is reduced because of a reduction in the potential energy. Therefore, starting from different initial conditions we obtain different positions where this jump occurs in decreasing fields. If we start from smaller values of the order parameter, the jump in magnetization moves towards smaller magnetic fields and consequently increases the hysteresis. The position of this jump can also be changed if we add random fluctuations of different magnitudes to the order parameter. The position given here corresponds to the field when the volume barrier disappears.

In conclusion, we showed that the hysteresis observed in mesoscopic disks [4], can be explained by considering meta-stability created by energy barriers: in the FwFD it is the BL surface barrier, whereas in the FrFD it is the volume barrier. In the ground state the magnetization of those disks is diamagnetic. Sweeping down the magnetic field brings the system in meta-stable states which have a substantial smaller diamagnetic behavior and can even be paramagnetic for certain thin disks. The size of the detector, has a substantial influence on the magnitude of the measured magnetization and can even change the sign of it.

This work is supported by the Flemish Science Foundation(FWO-Vl) grant No: G.0232.96, the European INTAS-93-1495-ext project, and the Belgian InterUniversity Attraction Poles (IUAP-VI). One of us (PSD) is supported by a post doctoral scholarship from the FWO-Vl and FMP is a Research Director with the FWOVl. Discussions with Dr. A. Geim is gratefully acknowledged.

* Electronic mail: deo@uia.ua.ac.be

** Permanent address: Institute of Theoretical and Applied Mechanics, Russian Academy of Sciences, Novosibirsk 
630090, Russia.

o Electronic mail: peeters@uia.ua.ac.be

[1] G. D. Cody and R. E. Miller, Phys. Rev. Lett. 16, 697 (1966), and references therein.

[2] A. S. Joseph and W. J. Tomasch, Phys. Rev. Lett. 12, 219 (1964); R. W. Deblois and W. DeSorbo, Phys. Rev. Lett. 12, 499 (1964).

[3] C. P. Bean and J. D. Livingston, Phys. Rev. Lett. 12, 14 (1964).

[4] A. K. Geim, I. V. Grigorieva, S. V. Dubonos, J. G. S. Lok, J. C. Maan, A. E. Filippov, and F. M. Peeters, Nature (London), 390, 259 (1997).

[5] P. S. Deo, V. A. Schweigert, F. M. Peeters, and A. K. Geim, Phys. Rev. Lett. 79, 4653 (1997); ibid Physica E 1, 297 (1997).

[6] V. A. Schweigert and F. M. Peeters, Phys. Rev. B 58, (1st June, 1998).

[7] D. J. Thompson, M. S. M. Minhaj, L. E. Wenger, and J. T. Chen Phys. Rev. Lett. 75, 529 (1995), and references therein.

[8] V. V. Moshchalkov, X. G. Qiu, and V. Bruyndoncx, Phys. Rev. B 55, 11793 (1997), and references therein.

[9] A. K. Geim, S. V. Dubonos, I. V. Grigorieva, J. G. S. Lok and J. C. Maan, Appl. Phys. Lett. 71, 2379 (1997).

[10] F. M. Peeters and X. Q. Li, Appl. Phys. Lett. 72, 572 (1998).

\section{FIGURE CAPTIONS}

Fig. 1. The magnetization (a) and dimensionless free energy in units of $H_{c}^{2} V / 8 \pi$ (b) for a FwFD (radius $R=0.8 \mu \mathrm{m}$, thickness $d=0.134 \mu \mathrm{m}$, coherence length $\xi(0)=0.183 \mu \mathrm{m}$ and penetration length $\lambda(0)=0.07 \mu \mathrm{m})$ as a function of magnetic field. The thin solid curves are the $2 \mathrm{D}$ solutions for different $L$ states. The ground state is given by the thick solid curve. The dotted and dashed curves are the increasing and decreasing field behavior obtained from the $3 \mathrm{D}$ solution.

Fig. 2. The experimental results for the magnetization (left scale) in increasing (circles) and decreasing (squares) magnetic field of the disk of Fig. 1. Theoretical results for the sample magnetization (right scale) are given by the dashed and dotted curves. Inclusion of the detector size, gives the corresponding thin and thick solid curves with reference to the left scale.

Fig. 3. The solid curve is the field distribution (applied field of $59.65 \mathrm{G}$ plus magnetic field due to the magnetization of the sample) along a line through the center of the disk of Fig. 1. The disk region and the detector region are indicated. The dotted curve is the corresponding curve at an applied field of $13 \mathrm{G}$ for a disk which is ten times thinner. The inset shows the magnetization versus decreasing applied field for this thin sample.

Fig. 4. The squares (circles) are the experimental data in increasing (decreasing) field for a FrFD (radius $R=0.5 \mu m$, thickness $d=0.15 \mu \mathrm{m}$, coherence length $\xi(0)=0.25 \mu \mathrm{m}$ and penetration length $\lambda(0)=0.07 \mu \mathrm{m})$. The dashed (solid) curve is the corresponding numerically calculated detector magnetization. 


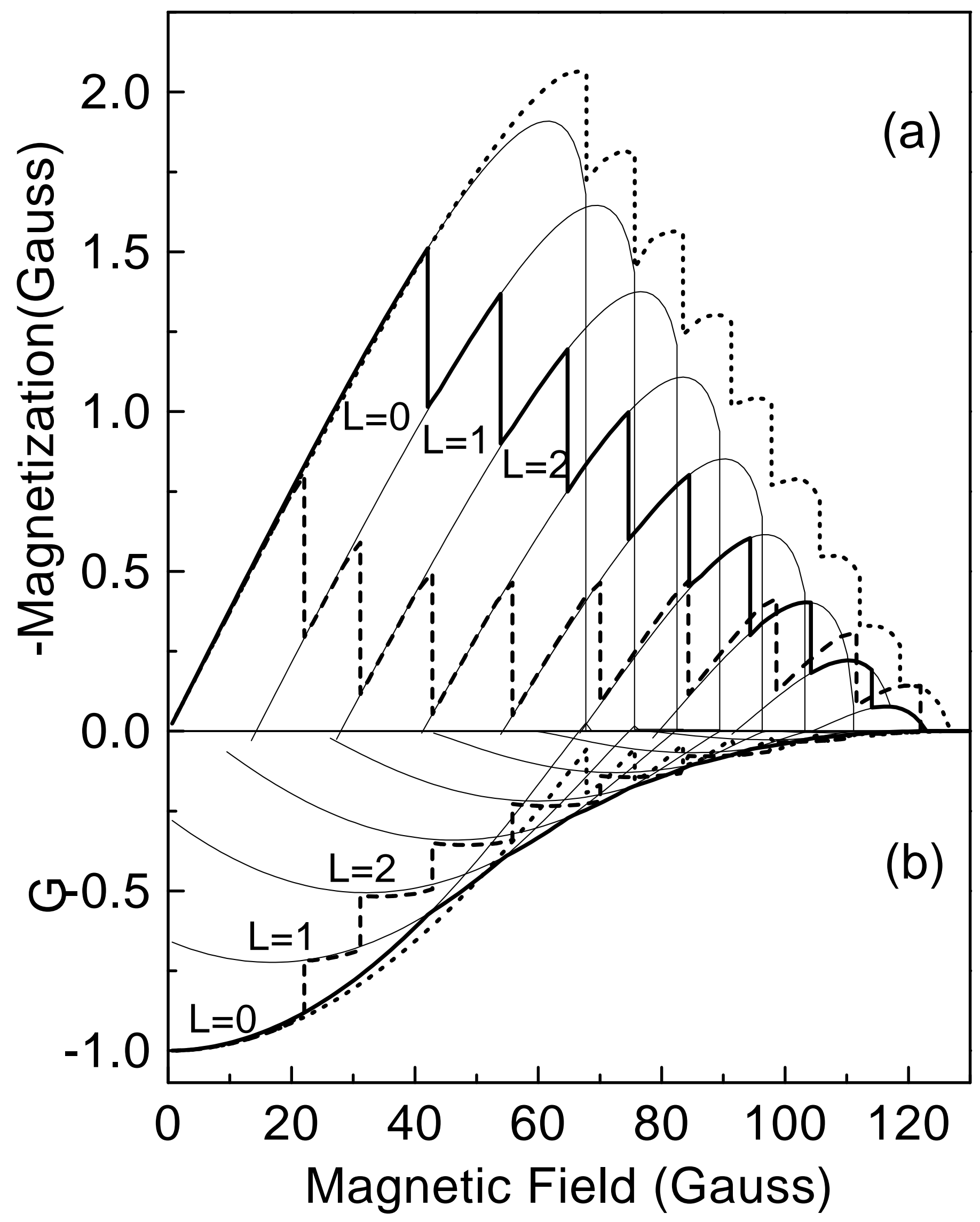




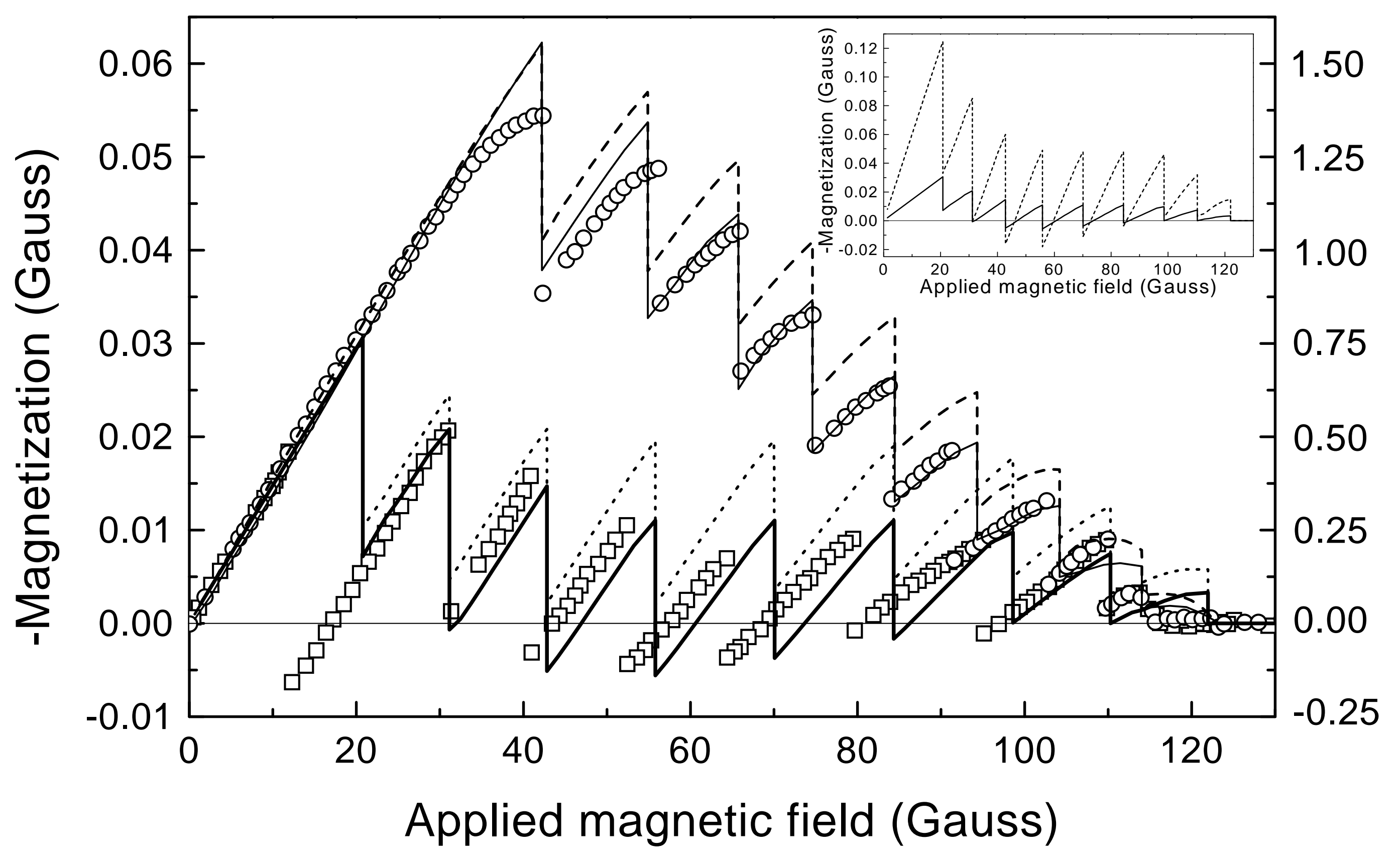




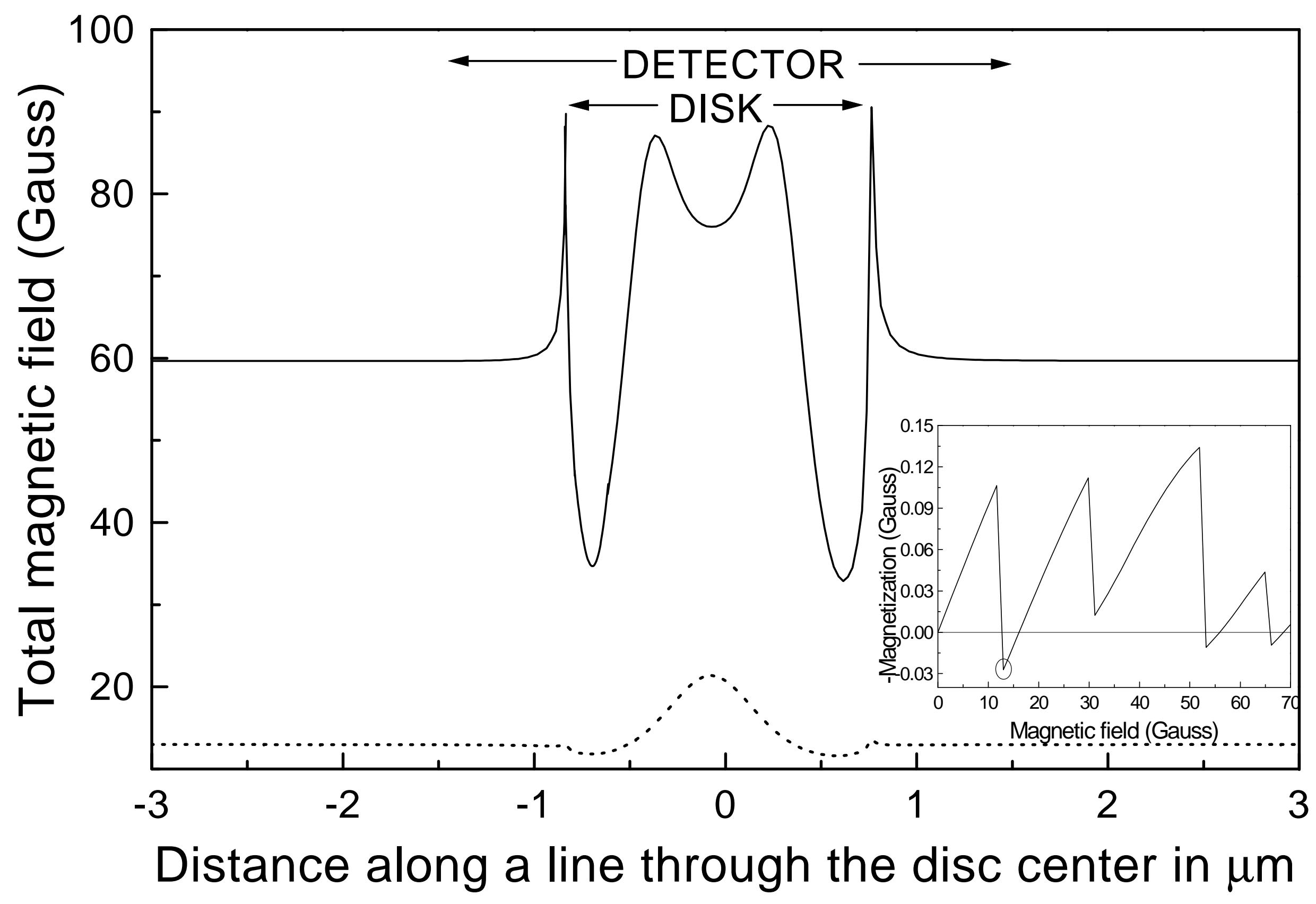




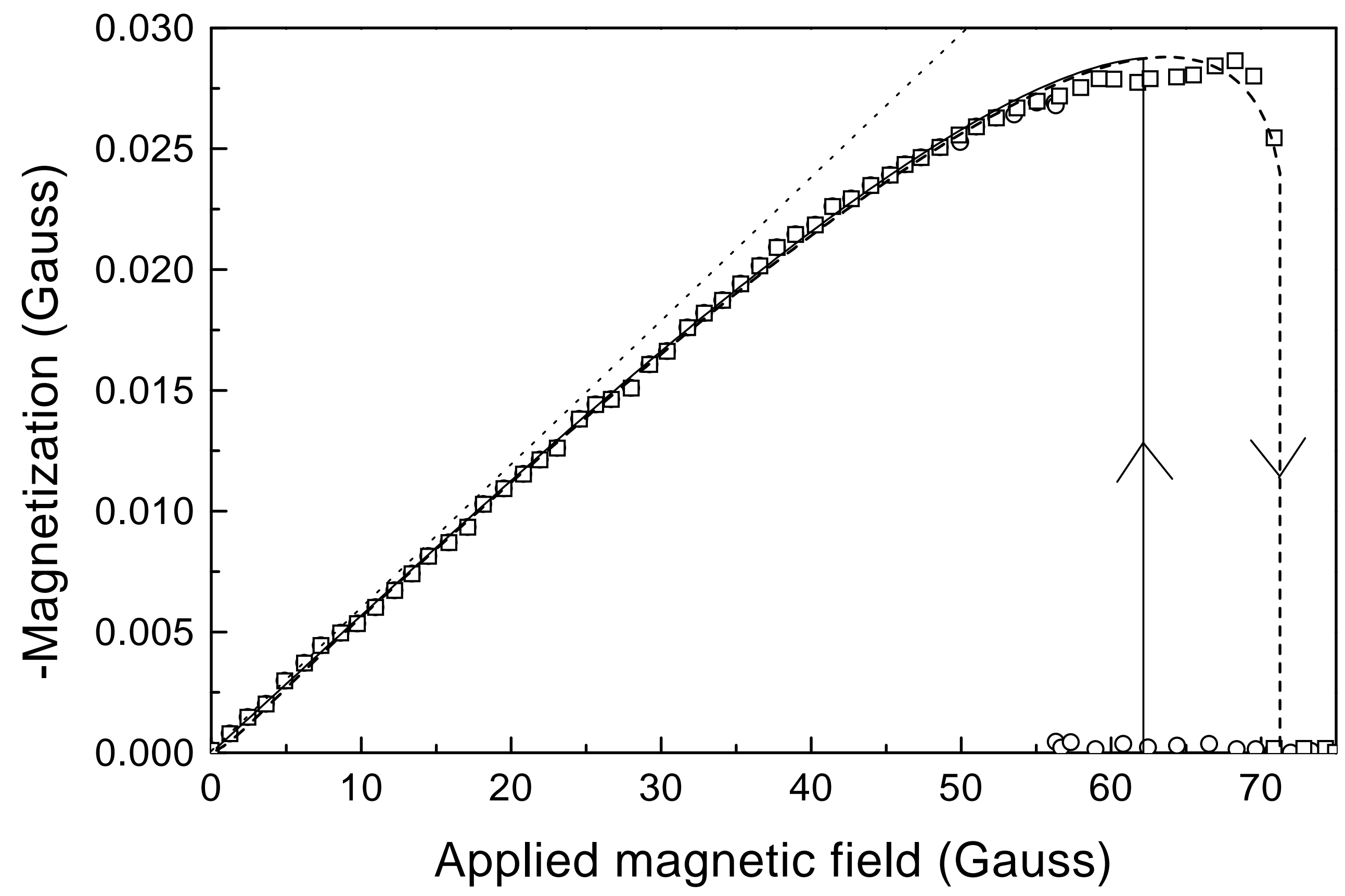

